\title{
PROPOSTA DE UMA NOVA ESTRATÉGIA DE CONTROLE PARAA FERMENTAÇÃO CERVEJEIRA
}

\section{PROPOSAL OF A NEW CONTROL STRATEGY FOR BEER FERMENTATION}

\author{
Diego Dias Carneiro* \\ Luiz Augusto da Cruz Meleiro**
}

\begin{abstract}
RESUMO
A cerveja ocupa uma importante posição no mercado econômico, pois é a bebida alcoólica mais consumida no Brasil e no mundo. Tendo em vista essa importância econômica, a busca por processos eficientes e com capacidade de manter a qualidade sensorial do produto final é recorrente nas cervejarias. Uma etapa importante do processo cervejeiro é a fermentação, pois é nessa fase que se formam os produtos e subprodutos do metabolismo das leveduras. Dessa forma, para a condução satisfatória do processo fermentativo, faz-se necessária a realização de estudos detalhados sobre a etapa fermentativa da produção de cerveja. Esse conhecimento permite analisar as principais variáveis que influenciam a fermentação e o modo como elas interagem, além de facilitar o desenvolvimento de estratégias de controle dessas variáveis. O objetivo desse trabalho é, por meio da modelagem matemática e da simulação computacional, propor uma nova estratégia de controle para a fermentação cervejeira. Através da simulação do modelo matemático do processo, verificou-se que o sistema de controle normalmente utilizado nas indústrias cervejeiras - que apresenta somente capacidade para refrigerar o tanque de fermentação - não é eficiente. Foi analisada a demanda energética para a implementação de um perfil de temperatura pré-determinado e confirmou-se a necessidade de um sistema que seja capaz não apenas de refrigerar o tanque de fermentação, mas também de aquecê-lo. A estratégia de controle proposta se mostrou eficiente, proporcionando ao operador a aplicação de perfis de temperatura que propiciem a condução satisfatória da fermentação cervejeira, fornecendo um produto final de qualidade para o consumidor.
\end{abstract}

Palavras-chave: Modelagem matemática. Fermentação cervejeira. Estratégia de controle.

\begin{abstract}
Nowadays, beer production plays an important role in the economic market, since it is the most consumed alcoholic beverage in Brazil and around the world. Considering this economic importance, the search for efficient production processes that are able to maintain the sensorial quality of the final product is a persistent challenge in breweries. Fermentation is an important stage of beer production process, since the products and by-products of the yeast metabolism
\end{abstract}

\footnotetext{
* Programa de Pós-Graduação em Ciência e Tecnologia de Alimentos - Universidade Federal Rural do Rio de Janeiro. E-mail: dieguinhode@yahoo.com.br

** Departamento de Tecnologia de Alimentos - Universidade Federal Rural do Rio de Janeiro. E-mail: meleiro@ufrj.br
} 
are formed in this step. To an appropriate conduction of the beer production, detailed studies on the fermentative process become more and more important. The resulting knowledge obtained from this research allows us to understand how the main process variables influence the fermentation, as well as the way they interact each other in order to develop a suitable control strategy. The mathematical modeling, together with the computational simulation, is a suitable tool for the study of the fermentative process. The objective of this work is to propose a new control strategy for the beer fermentation based on mathematical modeling and simulation of this process. Through the simulation of the process model, it was verified that the usually control system working on the breweries is only capable to refrigerate the fermentation vessel. The analysis of the energy demand for the control system track a temperature profile suggested that the thermal system must be able to both, refrigerate and heat the fermentation vessel. The proposed control strategy shows to be very efficient, providing the process operator the application of temperature profiles that lead to a satisfactory fermentation process and to a final product with the desired quality to the consumer.

Keywords: Mathematical modeling. Control strategy. Temperature profile.

\section{Introdução}

Atualmente a produção de bebidas alcoólicas contribui significantemente na economia de diversos países, onde a cerveja está entre uma das mais importantes (HUI, 2006). Essa informação é confirmada por SENAD (2007) e por Nelson (2005), que citam, respectivamente, que a cerveja é a bebida alcoólica mais consumida no Brasil e no mundo, perdendo em popularidade apenas para a água e para o chá. Dados do SINDCERV em 2005 mostram que o Brasil ocupa o $9^{\circ}$ lugar no quesito consumo per capita de cerveja, com o valor de 47 litros/ano.

Essa importância econômica leva, cada vez mais, à busca por estudos que tornem o processo cervejeiro mais barato, mais rápido e com qualidade. Nesse processo, as propriedades físico-químicas e sensoriais do produto final devem ser mantidas dentro de padrões pré-estabelecidos.

Dentre os estágios que compreendem o processo de produção de cerveja, a etapa fermentativa foi escolhida como objeto de estudo desse trabalho, pois é uma etapa que exige o controle mais rígido do processo e onde, através do metabolismo microbiano, são formados os produtos e subprodutos que caracterizam a cerveja.

Segundo Rosenstock \& Coimbra (1999), a fermentação cervejeira, de uma forma simples, consiste na conversão dos açúcares fermentescíveis do mosto em álcool, gás carbônico e subprodutos pela ação da levedura. O principal foco dessa etapa é, usando a tecnologia adequada, conduzir as interações de todos os parâmetros envolvidos no processo (composição do mosto, linhagem da levedura, temperatura de processo, geometria do tanque, etc.), para obter a cerveja com as características sensoriais, químicas e físico-químicas previamente determinadas (BRIGGS, 2004).

Uma metodologia utilizada com frequência para verificar como os parâmetros envolvidos na fermentação interagem, permitindo um melhor controle do processo, é a modelagem matemática aliada à simulação computacional.

Foram encontrados na literatura poucos modelos matemáticos que representem a etapa fermentativa da produção de cerveja. Para o desenvolvimento dessa pesquisa foram selecionados três modelos fenomenológicos (GEE, 1990; ANDRÉS-TORO, 1998; TRELEA et al., 2004), escolhidos com base na sua validação experimental e nas variáveis de processo utilizadas no modelo.

O modelo escolhido para elaborar uma nova estratégia de controle para a fermentação cervejeira, principal objetivo desse trabalho, foi o proposto por Gee (1990), pois dentre os selecionados é o único que apresenta o balanço de energia para o tanque de fermentação, que é a característica necessária para a aplicação de uma estratégia de controle. 


\section{Materiais e métodos}

O modelo matemático utilizado no trabalho ora apresentado para simular o processo fermentativo foi o desenvolvido por Gee (1990). O autor construiu o referido modelo baseado nos estudos de Fidget \& Smith (1977) e de Engasser et al. (1981), nos quais as melhores características de cada um desses modelos foram utilizadas. O autor validou e ajustou os parâmetros das equações do modelo através da realização de fermentações do mosto cervejeiro em laboratório e em planta piloto, conduzido-as em diferentes condições de processo.

As equações diferenciais do modelo utilizadas nesse trabalho foram as que descrevem as seguintes dinâmicas:

Consumo de glicose: $\frac{d G}{d t}=-\mu_{1} X$

Consumo de maltose: $\frac{d M}{d t}=-\mu_{2} X$

Consumo de maltotriose: $\frac{d N}{d t}=-\mu_{3} X$

Parâmetro Cinético 1: $\mu_{1}=\frac{\mu_{G} G}{K_{G}+G}$

Parâmetro Cinético 2: $\mu_{2}=\frac{\mu_{M} M}{K_{M}+M} \frac{K_{G}^{\prime}}{K_{G}^{\prime}+G}$

Parâmetro Cinético 3:

$$
\mu_{3}=\frac{\mu_{N} N}{K_{N}+N} \frac{K_{G}^{\prime}}{K_{G}^{\prime}+G} \frac{K_{M}^{\prime}}{K^{\prime}{ }_{M}+M}
$$

Crescimento de biomassa:

$$
\frac{d X}{d t}=\left(Y_{X G} \mu_{1}+Y_{X M} \mu_{2}+Y_{X N} \mu_{3}\right) \frac{K_{X}^{\prime}}{K_{X}^{\prime}+\left(X-X_{0}\right)} X
$$

Produção de Etanol:

$$
\frac{d E}{d t}=-Y_{E G} \frac{d G}{d t}-Y_{E M} \frac{d M}{d t}-Y_{E N} \frac{d N}{d t}
$$

Produção de $\mathrm{CO}_{2}$ :

$$
\left\{\begin{array}{l}
\frac{d C_{l}}{d t}=\left\{\begin{array}{c}
K_{G L}\left(C_{s a t}-C_{l}\right) \quad C_{l}<C_{s a t} \\
0 \quad C_{l}=C_{s a t}
\end{array}\right. \\
\frac{d C_{g}}{d t}=-Y_{C G} \frac{d G}{d t}-Y_{C M} \frac{d M}{d t}-Y_{C N} \frac{d N}{d t}-K_{G L}\left(C_{s a t}-C_{l}\right)
\end{array}\right.
$$

Balanço de Energia:

$$
\frac{d T}{d t}=\frac{\Delta H_{F}}{\rho_{F} V_{F} C p_{F}}-\frac{h A_{C}}{\rho_{F} V_{F} C p_{F}}\left(T-T_{C}\right)
$$

As dinâmicas dependentes da temperatura de condução de processo têm suas taxas alteradas de acordo com a função de Arrhenius:

$P_{i}=P_{i 0} \exp \left[\frac{-E_{A_{i}}}{R(T+273,15)}\right]$

onde $P$ corresponde a uma das constantes de Michaelis utilizadas nas equações do modelo $(\mu, K$ ou $K^{\prime}$ ); $i$ representa o substrato utilizado, ou seja, glicose $(G)$, maltose $(M)$ ou maltotriose $(N) ; E_{A}$ é a energia de ativação referente a cada parâmetro; $R$ é a constante cinética dos gases; e $T$ a temperatura do processo, em ${ }^{\circ} \mathrm{C}$.

Uma importante característica desse modelo é o desenvolvimento do balanço de energia para o tanque de fermentação, que permite investigar estruturas de controle mais próximas da realidade, conforme ilustra a Figura 1.

O software utilizado para resolver esse conjunto de equações diferenciais foi o SciLab® 5.1.1, no qual a integração numérica das equações diferenciais ordinárias que representam o processo foi feita utilizando o método de Runge-Kutta de $4^{\mathrm{a}}$ ordem. 
Figura 1 - Configuração básica do sistema de controle.

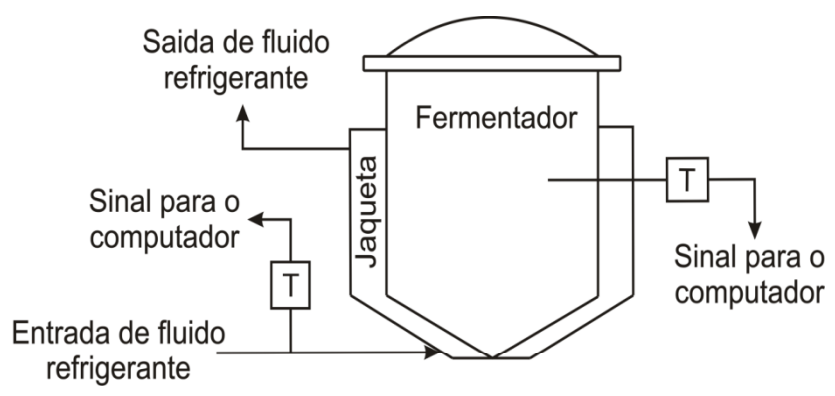

A elaboração da nova estratégia de controle para essa fermentação foi idealizada a partir de observações do processo proposto por Gee (1990) ilustrado na Figura 1 - em que o autor utiliza um sistema de controle capaz apenas de refrigerar a temperatura do tanque, idêntico ao sistema de controle utilizado atualmente nas indústrias cervejeiras. Essa estratégia, apesar de ser utilizada nas indústrias, não é muito eficaz para fins de otimização do proces-

Figura 2 - Perfil de temperatura de fermentação proposto por Andrés-Toro et al. (1998).

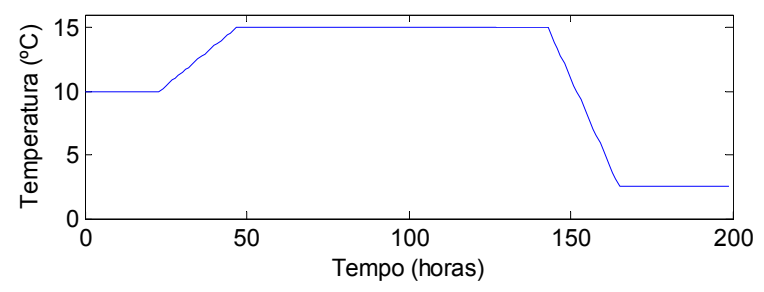

\section{Resultados e discussões}

As condições iniciais utilizadas para realizar a simulação computacional do modelo são apresen- so, visto que para acompanhar perfis de temperatura mais elaborados, como o descrito por Andrés-Toro et al. (1998) e apresentado na Figura 2, é necessário um sistema de controle que também seja capaz de aquecer o mosto. Mesmo sendo a fermentação um processo exotérmico, o calor gerado não é suficiente para seguir perfis que necessitem de taxas de aquecimento elevadas, fato que será demonstrado nos resultados apresentados a seguir.

Para verificar a necessidade de uma nova estratégia de controle para a fermentação cervejeira, foi imposto ao processo um perfil de temperatura semelhante aos perfis empregados atualmente no desenvolvimento de estratégias de otimização para essa fermentação, conforme ilustrado na Figura 3. Com o perfil proposto é possível investigar a demanda térmica necessária para a sua aplicação na fermentação do mosto.

Figura 3 - Perfil de temperatura proposto para investigação da demanda térmica da fermentação.

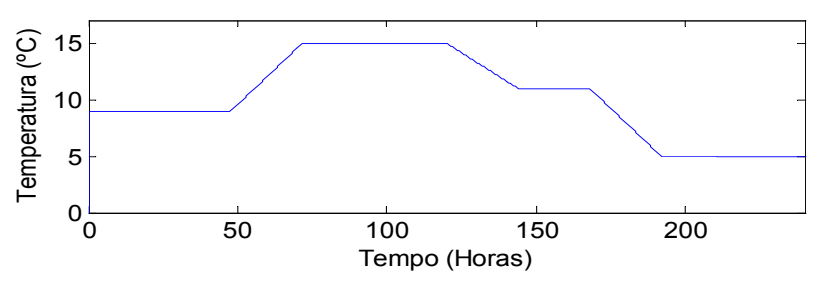

tadas na Tabela 1, e o resultado da simulação é ilustrado na Figura 4.

Tabela 1 - Condições iniciais do processo fermentativo.

\begin{tabular}{ccccccc}
\hline Glicose & Maltose & Maltotriose & Biomassa & Etanol & $\boldsymbol{C O}_{2}$ & Temperatura \\
\hline $70 \mathrm{~mol} / \mathrm{m}^{3}$ & $220 \mathrm{~mol} / \mathrm{m}^{3}$ & $40 \mathrm{~mol} / \mathrm{m}^{3}$ & $170 \mathrm{~mol} / \mathrm{m}^{3}$ & $0 \mathrm{~mol} / \mathrm{m}^{3}$ & $0 \mathrm{~mol} / \mathrm{m}^{3}$ & $8^{\circ} \mathrm{C}$ \\
\hline
\end{tabular}


Figura 4 - Resultado da simulação computacional do modelo do processo proposto por Gee (1990).
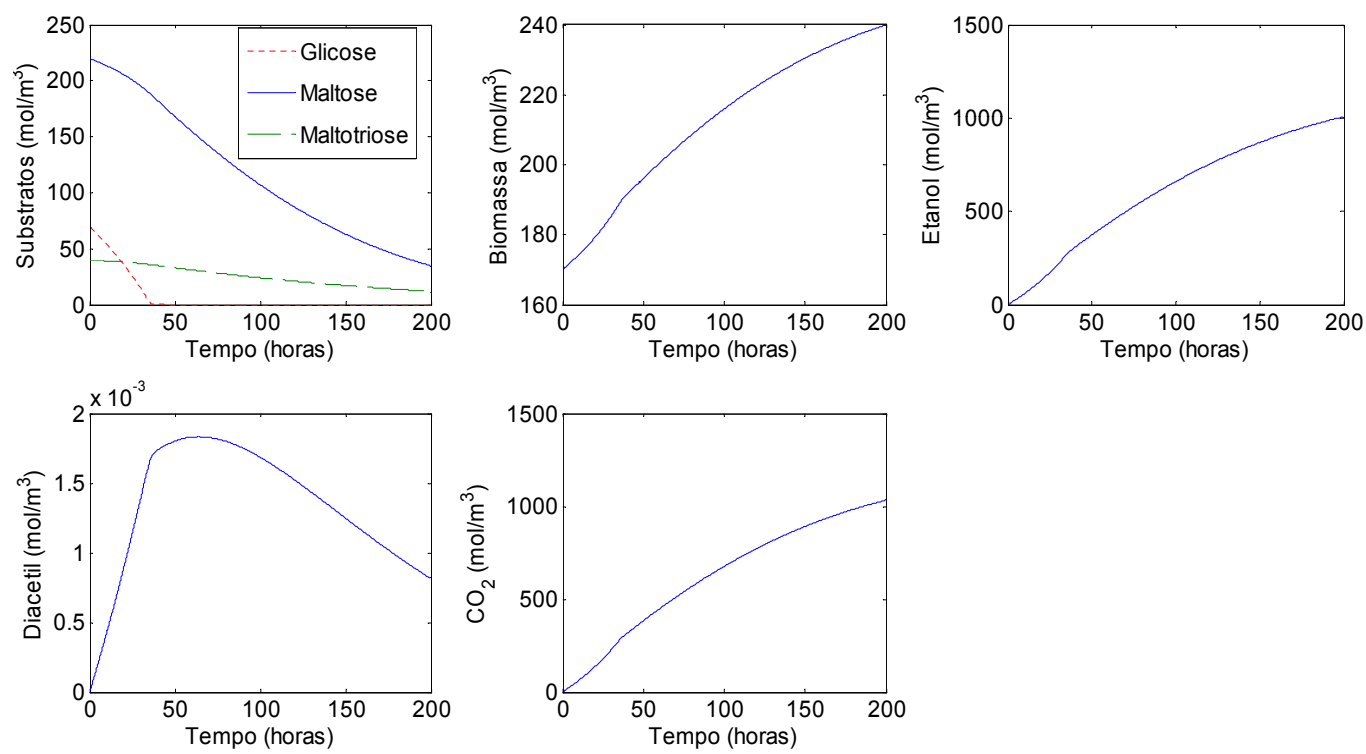

O comportamento dinâmico das variáveis de processo estudadas (Figura 4), assim como seus valores finais, apresentara $\mathrm{m}$ conformidade com os resultados apresentados no trabalho original de Gee (1990), Tal fato indica que os resultados obtidos da

resolução numérica e implementados neste trabalho estão corretos e, portanto, esses resultados representam adequadamente o comportamento dinâmico da planta de fermentação cervejeira estudada.

Figura 5 - Dinâmica da temperatura e da taxa de refrigeração fermentativo: Gee (1990).

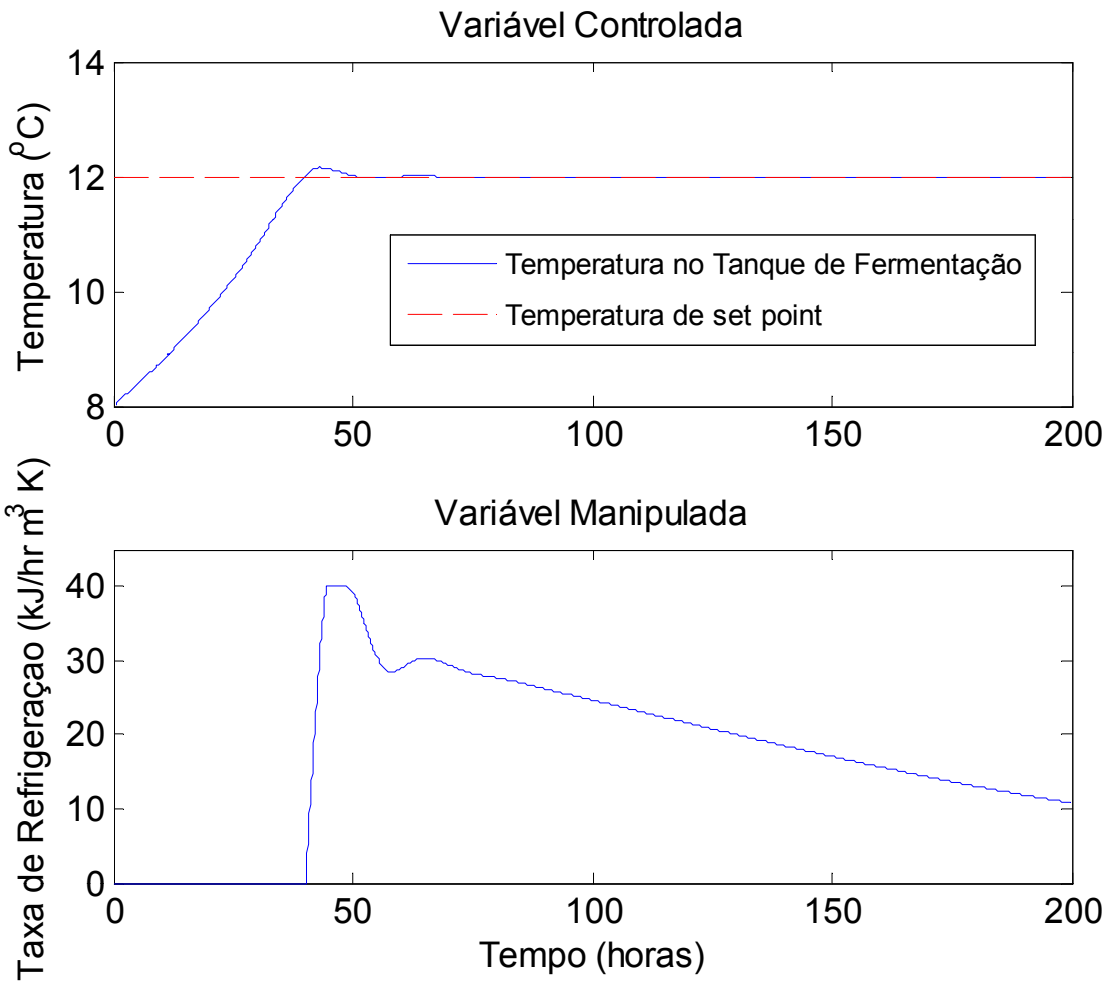


O resultado do balanço de energia é apresentado na Figura 5, onde se pode observar o comportamento dinâmico da temperatura de fermentação e a temperatura máxima na qual o processo fermentativo deve ser conduzido, assim como as ações de controle impostas ao processo através da manipulação da taxa de refrigeração. Quando a temperatura do sistema atinge o valor máximo $\left(12^{\circ} \mathrm{C}\right)$ determinado por Gee (1990), o controlador é acionado para mantê-la no set-point até o final da fermentação. $\mathrm{O}$ comportamento dinâmico da temperatura de fermentação e as ações do controlador apresentadas na Figura 5 também apresentam conformidade com o trabalho do referido autor.

É importante ressaltar que a fase de aquecimento apresentada na Figura 5 é proporcionada pela própria fermentação, que é um processo exotérmico, e não por qualquer meio externo. O controlador é responsável apenas por evitar que a temperatura de fermentação ultrapasse o set-point.

O fermentador com o sistema de controle apresentado por Gee (1990) não é capaz de acompanhar perfis de temperatura que apresentem uma rampa acentuada de aquecimento do processo, pois o calor fornecido pela fermentação não é suficiente para promover um rápido aumento de temperatura, conforme ilustra a Figura 6. O fato de o calor proveniente do metabolismo dos substratos não ser suficiente para aquecer o mosto na taxa desejada, demonstra que o sistema de controle utilizado nas indústrias cervejeiras não é adequado para tal tarefa. A demanda energética necessária para acompanhar perfis de temperatura com tais características é ilustrada na Figura 7.

Figura 6 - Dinâmica da temperatura de fermentação com controle de temperatura utilizando apenas fluido refrigerante.

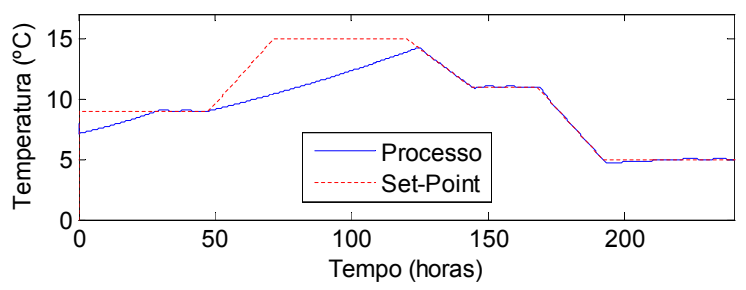

Figura 7 - Demanda energética para o perfil de temperatura proposto.

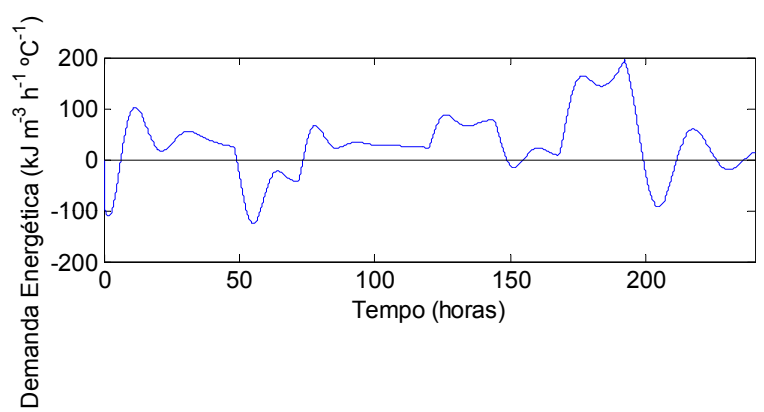

Observando a Figura 7, verifica-se que para a temperatura de fermentação acompanhar o perfil estabelecido, é necessário um sistema de controle capaz de aquecer e refrigerar o tanque de fermentação.

A maior parte das estruturas de controle que trabalham com sistema de aquecimento e resfriamento utiliza o controle em cascata como estratégia de controle (CHYLLA; HAASE, 1993; BEQUETTE, 2002; MADAR et al., 2004). Essa estratégia, apesar da sua eficácia, exige um sistema com dois controladores, fato que exige da indústria um maior recurso financeiro e operacional, pois tal sistema apresenta um maior número de parâmetros para serem ajustados.

Visando a simplificar essa estrutura de controle, é apresentada uma proposta de estratégia de controle para o processo fermentativo que exige menos recursos tecnológicos e operacionais para o seu funcionamento, pois utiliza apenas um controlador.

Essa proposta consiste na manipulação de um único fluido térmico (água) através da jaqueta do fermentador com chaveamento entre ciclos de refrigeração e aquecimento. Nela, o aquecimento do fluido térmico é realizado através de um trocador de calor (Figura 8). 
Figura 8 - Proposta da estrutura de controle para fermentação cervejeira.

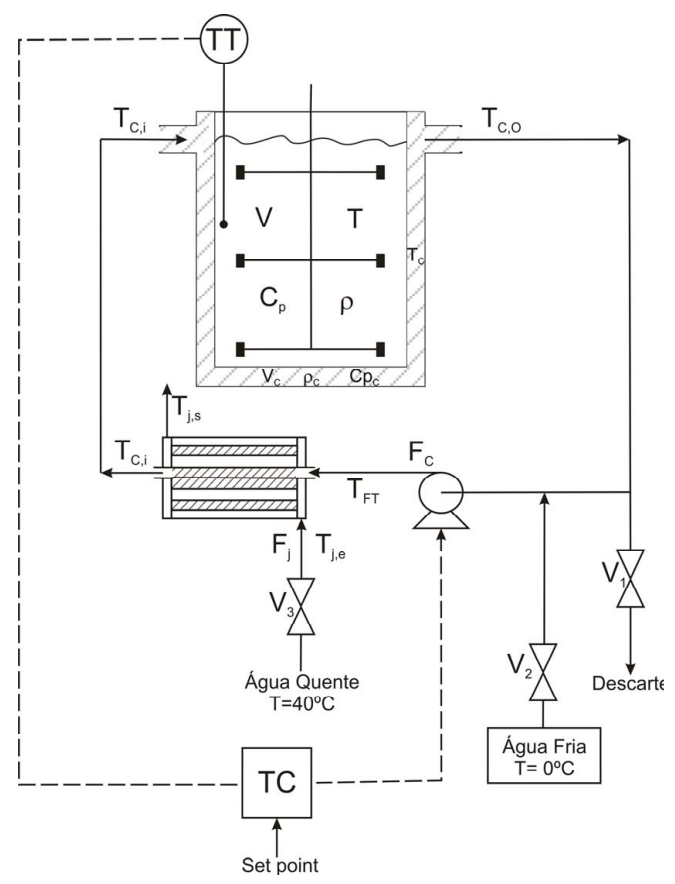

A estratégia de chaveamento que determina se o sistema será resfriado ou aquecido é demonstrada na Tabela 2 .

Tabela 2 - Condições de operação dos ciclos de aquecimento e resfriamento.

\begin{tabular}{ccc}
\hline Equipamento & Resfriamento & Aquecimento \\
\hline $\boldsymbol{V}_{1}$ & Aberta & Fechada \\
$\boldsymbol{V}_{2}$ & Aberta & Fechada \\
$\boldsymbol{V}_{3}$ & Fechada & Aberta \\
$\boldsymbol{F}_{j}$ & $0 \mathrm{~m}^{3} / \mathrm{h}$ & $0,16 \mathrm{~m}^{3} / \mathrm{h}$ \\
$\boldsymbol{T}_{\boldsymbol{F} T}$ & $0{ }^{\circ} \mathrm{C}$ & $T_{C, O}$ \\
\hline
\end{tabular}

Nessa tabela, $V_{1}$ é a válvula de descarte do fluido térmico, $V_{2}$ é a válvula que alimenta o sistema com água gelada, $V_{3}$ é a válvula que alimenta o trocador de calor com água quente $\left(40^{\circ} \mathrm{C}\right), F_{j}$ é a vazão de água quente no trocador de calor e $T_{F T}$ é a temperatura do fluido térmico.

O dimensionamento utilizado para o tanque de fermentação foi apresentado por GEE (1990):

Tabela 3 - Dados do reator (GEE, 1990).

\begin{tabular}{cc}
\hline \multicolumn{2}{c}{ Dados do Fermentador } \\
\hline $\boldsymbol{V}$ & $0,235 \mathrm{~m}^{3}$ \\
$\boldsymbol{\rho}$ & $1040 \mathrm{~kg} / \mathrm{m}^{3}$ \\
$\boldsymbol{C}_{\boldsymbol{P}}$ & $4,016 \mathrm{~kJ} \mathrm{~kg}^{-1} \mathrm{C}^{-1}$ \\
$\boldsymbol{h}$ & $879 \mathrm{~kJ} \mathrm{~h}^{-1} \mathrm{~m}^{-2} \mathrm{C}^{-1}$ \\
\hline
\end{tabular}

Tabela 4 - Dados da jaqueta (GEE, 1990).

\begin{tabular}{cc}
\hline \multicolumn{2}{c}{ Dados da Jaqueta } \\
\hline $\boldsymbol{V}_{\boldsymbol{C}}$ & $0,032 \mathrm{~m}^{3}$ \\
$\boldsymbol{\rho}_{C}$ & $1062 \mathrm{~kg} / \mathrm{m}^{3}$ \\
$\boldsymbol{C}_{P C}$ & $3,768 \mathrm{~kJ} \mathrm{~kg}^{-1}{ }^{\circ} \mathrm{C}^{-1}$ \\
$\boldsymbol{A}_{\boldsymbol{C}}$ & $1,746 \mathrm{~m}^{2}$ \\
\hline
\end{tabular}

O dimensionamento do trocador de calor foi baseado nos dados de Andrietta (1994). Segundo o autor, o volume estimado do trocador de calor deveria ser:

$$
V_{j}=V_{T_{C}}=0,024 m^{3} \quad(10 \% \text { de } V)
$$

Contudo, como o volume da jaqueta é $V_{C}=0,032 m^{3}$, decidiu-se optar por:

$$
V_{j}=V_{T_{C}}=V_{C}=0,032 \mathrm{~m}^{3}
$$

Dessa forma, segundo a heurística adotada por Andrietta (1994), de utilizar trocadores de calor com pelo menos $10 \%$ do volume do reator, garante-se que os dois sistemas de troca térmica apresentem volume suficiente para garantir uma boa troca térmica.

O fermentador utilizado no trabalho de Andrietta (1994) apresenta volume $V=210 \mathrm{~m}^{3}$. Os dados do trocador de calor utilizado no mesmo trabalho são apresentados na Tabela 5.

Tabela 5 - Dados do trocador de calor (Andrietta, 1994).

\begin{tabular}{ccc}
\hline & \multicolumn{2}{c}{ Trocador de Calor } \\
\hline & Casco & Tubo \\
\hline Volume & $\left(V_{j}\right) 20 \mathrm{~m}^{3}$ & $\left(\mathrm{~V}_{\mathrm{C}}\right) 20 \mathrm{~m}^{3}$ \\
Área de Troca & $76 \mathrm{~m}^{2}$ & $76 \mathrm{~m}^{2}$ \\
Vazão & $\left(F_{j}\right) 400 \mathrm{~m}^{3} / \mathrm{h}$ & $\left(\mathrm{F}_{\mathrm{C}}\right) 400 \mathrm{~m}^{3} / \mathrm{h}$ \\
\hline
\end{tabular}

O volume do trocador de calor utilizado corresponde a aproximadamente $10 \%$ do volume do fermentador. Nesse caso, o trocador de calor apresenta uma relação entre $F_{j}$ e $V_{j,}$ na qual:

$$
\frac{F_{j}}{V_{j}}=20 h^{-1}
$$

Ou seja, o volume de fluido refrigerante é renovado 20 vezes a cada hora, ou uma vez a cada 3 minutos. Como estimativa inicial, foi adotada a mesma relação para o trocador proposto, onde: 


$$
F_{j}=20 V_{j}=20 \times 0,032=0,64 \frac{m^{3}}{h} \cong 11 \frac{L}{\min }
$$

Outra opção é usar a vazão máxima de circulação de fluido térmico proposta por GEE (1990), na qual:

$$
F_{j}=F_{C, \max } \cong 0,16 \frac{m^{3}}{h} \cong 2,7 \frac{L}{\min }
$$

A relação entre o volume e a área de troca térmica no trocador de calor de Andrietta (1994) é:

$$
\frac{A_{T C}}{V_{j}}=3,8 m^{-1}
$$

Adotando essa relação para o trocador de calor proposto:

$$
A_{T C}=3,8 V_{j}=0,1216 m^{2}
$$

O balanço de energia do fermentador é dado por (GEE, 1990):

Tanque: $\frac{d T}{d t}=\frac{\Delta H_{F}}{\rho_{F} V_{F} C p_{F}}-\frac{h A_{C}}{\rho_{F} V_{F} C p_{F}}\left(T-T_{C}\right)$

Jaqueta: $\frac{d T_{C}}{d t}=\frac{2 F_{C}}{\rho_{C} V_{C}}\left(T_{i n}-T_{C}\right)+\frac{h A_{C}}{\rho_{C} V_{C} C p_{C}}\left(T-T_{C}\right)$

O balanço de energia para o trocador de calor é baseado no apresentado por Andrietta (1994):

Água Quente:

$$
\frac{d T_{j}}{d t}=\frac{F_{j}}{V_{j}}\left(T_{j, e}-T_{j}\right)+\frac{U A}{\rho_{j} V_{j} C p_{j}}\left(T_{j}-T_{C, i}\right)
$$

Fluido Térmico:

$$
\frac{d T_{C, i}}{d t}=\frac{F_{C}}{V_{C}}\left(T_{F T}-T_{C, i}\right)+\frac{U A}{\rho_{T C} V_{T C} C p_{T C}}\left(T_{j}-T_{C, i}\right)
$$

A densidade e o calor específico do fluido térmico foram considerados iguais aos valores adotados por Gee (1990).
Para verificar a eficiência e a eficácia do controlador proposto, foi feita uma simulação computacional do modelo proposto por Gee (1990) utilizando o perfil de temperatura proposto (Figura 3). É importante ressaltar que o intuito é apresentar a eficiência do controlador para o acompanhamento satisfatório do perfil proposto, pois esse perfil foi proposto com base em outros modelos (ANDRÉS-TORO et al., 1998). Para otimizar o modelo proposto por Gee (1990) seria necessário realizar um estudo de otimização de temperatura para o referido modelo. Os resultados obtidos na simulação do processo com o perfil de temperatura proposto são apresentados abaixo:

Figura 9 - Temperatura no tanque de fermentação: perfil proposto.

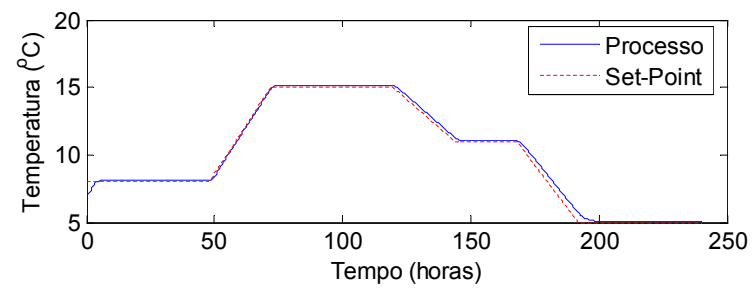

Figura 10 - Vazão de fluido térmico: perfil proposto.

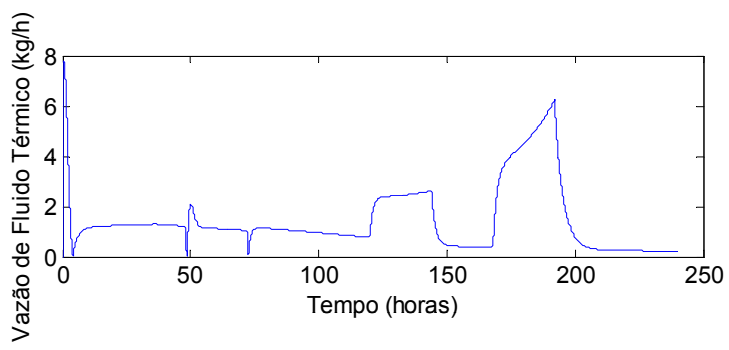


Figura 11 - Aberturas das válvulas do fluido de refrigeração: perfil proposto.

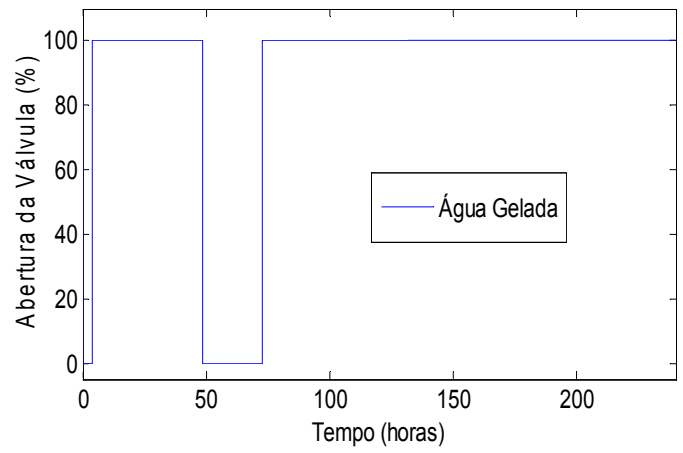

A partir de observações das figuras acima, verifica-se que a estratégia de controle proposta apresentou resultados satisfatórios, pois foi capaz de manter a temperatura do tanque de fermentação muito próxima da temperatura do perfil até o final do processo. Além disso, a variável controlada não apresentou oscilações bruscas e o chaveamento

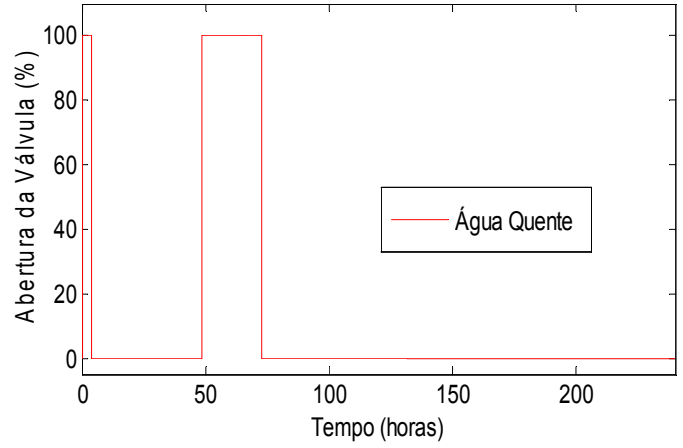

responsável pelo controle do aquecimento ou resfriamento do processo não foi utilizado de forma excessiva, observando-se apenas duas operações de abertura/fechamento das válvulas. Os comportamentos dinâmicos da concentração de substratos, biomassa, etanol $\mathrm{CO}_{2}$ e diacetil são apresentados a seguir:

Figura 12 - Dinâmica da concentração das variáveis de processo: perfil proposto.
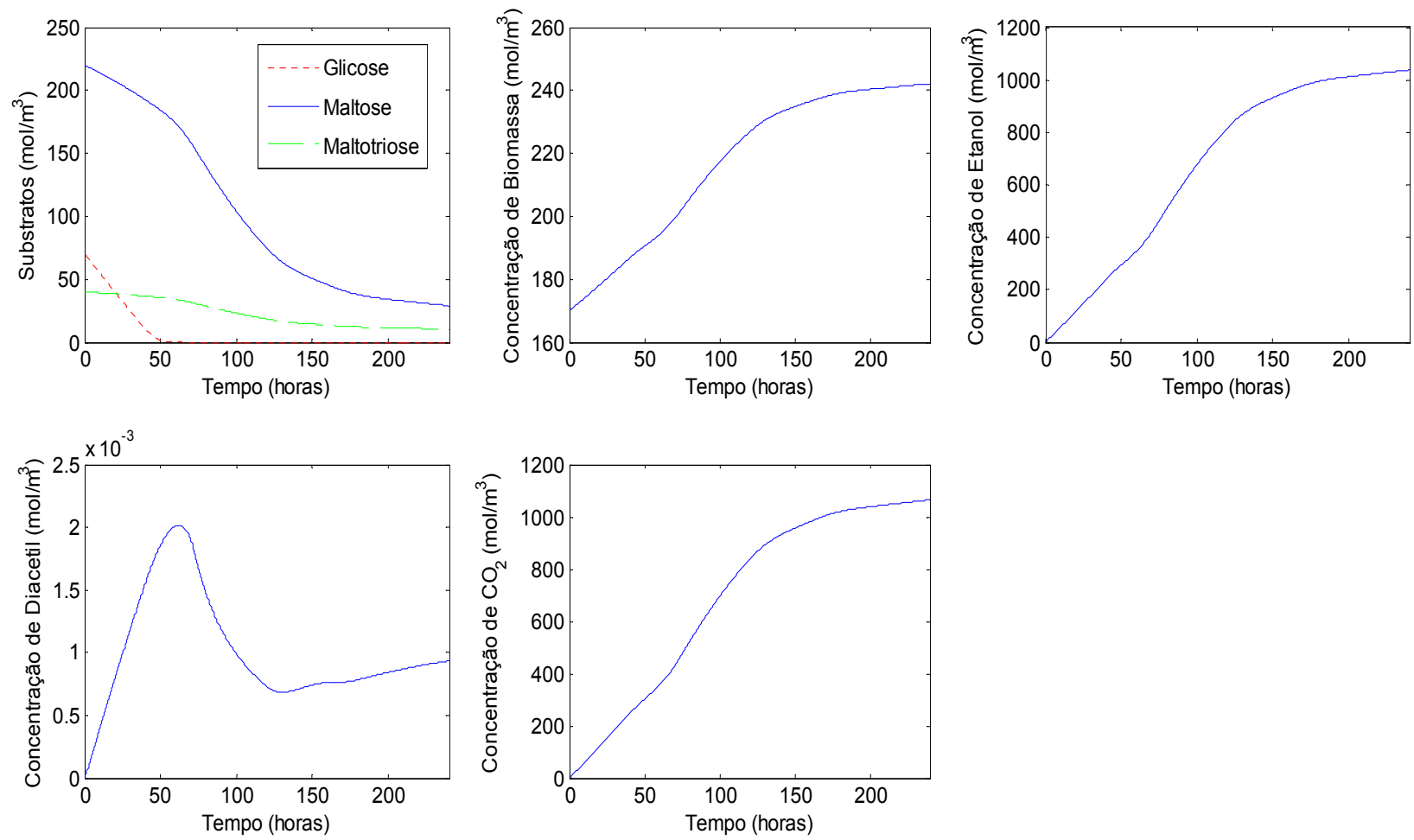

Com exceção da dinâmica da concentração de diacetil, as demais variáveis apresentaram comportamento similar às apresentadas por Gee (1990) e com concentração final bem próxima. A concentração de diacetil aumenta quando o processo é resfriado de $15^{\circ} \mathrm{C}$ até uma temperatura de $5^{\circ} \mathrm{C}$, resfriamento esse que ocorre na segunda metade do processo. Pode-se inferir, de acordo com o modelo matemático que descreve o comportamento do diacetil, que no momento em que o resfriamento ocorre a parcela responsável pela produção de diacetil assume valor maior do que a parcela responsável pelo seu consumo. 


\section{Conclusões}

A investigação da demanda térmica necessária para manter o processo cervejeiro sob um perfil de temperatura diferente do perfil utilizado industrialmente foi uma ferramenta essencial para o desenvolvimento de uma nova estratégia de controle do processo. Através dessa investigação, foi possível verificar que as estratégias de controle normalmente utilizadas (que apenas refrigeram o fermentador) não são adequadas para a implementação de perfis ótimos de temperatura, visto que o calor produzido no processo fermentativo não é suficiente para elevar a temperatura do mosto nas taxas impostas por tais perfis. A partir dessas observações, foi possível elaborar uma estratégia de controle relativamente simples, que envolve a inserção de um trocador de calor e três válvulas de regulagem de vazão, para resolver um problema de controle com soluções potencialmente complexas.

Através de estudos de simulação do processo cervejeiro realizados com um modelo validado industrialmente, a estratégia de controle proposta neste trabalho mostrou-se capaz de conduzir de forma satisfatória o processo fermentativo, sendo capaz de manter a temperatura do tanque de fermentação muito próxima do perfil de temperatura proposto para esse processo durante todo o tempo de processamento.

Com essa estratégia de controle é possível aplicar perfis ótimos de temperatura que permitam atingir diversos benefícios para o processo, como, por exemplo, a redução do tempo de fermentação com a manutenção das características sensoriais da cerveja.

\section{Referências}

ANDRÉS-TORO B. et al. A kinetic model for beer production under industrial operational conditions. Mathematics and Computers in Simulation, v. 48, p. 65-74, 1998.

ANDRÉS-TORO, B.; GÍRON-SIERRA, J. M.; FERNANDEZ-BLANCO, P.; LÓPEZ-OROZCO, J. A.; BESADA-PORTAS, E. Multiobjective optimization and multivariable control of the beer fermentation process with the use of evolutionary algorithms. Journal of the Zhejiang University - SCIENCE, v. 5, n. 4, p.378-389, 2004.

ANDRIETTA, S. R. Modelagem, simulação e controle de fermentação alcoólica contínua em escala industrial.
Tese de Doutorado, Universidade Estadual de Campinas Faculdade de Engenharia de Alimentos - FEA - UNICAMP, Campinas -SP - Brasil.

BEQUETTE, B. W. Behavior of a CSTR with a recirculating jacket heat transfer system. Proceedings of the American Control Conference, p.8-10, 2002.

BRIGGS, D. E. Brewing science and practice. Woodhead Publishing, 2004.

CHYLLA, R. W.; RANDALL HAASE, D. Temperature control of semibatch polymerization reactors. Computer Chem. Eng., v. 17, n. 3, p.257-264, 1993.

GEE, D. A. Modelling, optimal control, state estimation, and parameter identification applied to a batch fermentation process. Tese de Ph.D., University of Colorado, 1990.

HUI, Y. H. Food biochemistry and food processing. Blackwell Publishing, 2006.

MADAR, J. et al. Tendency model-based improvement of the slave loop in cascade temperature control of batch process units. Computers \& Chemical Engineering, v.28, n. 5 , p.737-744, 2004.

NELSON, M. The barbarian's beverage: a history of beer in Ancient Europe. Routledge, 2005.

ROSENSTOCK, T. R.; COIMBRA, V. S. Cerveja. 1999. In: Universidade Federal de São Paulo - Escola Paulista de Medicina. Disponível em: <http://www.virtual.epm.br/ material/tis/curr-bio/trab99/alcool/index.htm>. Acesso em: 5 dez. 2008.

SENAD - Secretaria Nacional Antidrogas. Cerveja é a bebida mais consumida no Brasil. Disponível em: <www. senad.gov.br/releases/Cerveja_Brasil.pdf>. Acesso em: 21 fev. 2009.

TRELEA, I. C.; TITICA, M.; CORRIEU, G. Dynamic optimisation of the aroma production in brewing fermentation. Journal of Process Control., v. 14 , p. 1-16, 2004.

Recebido para publicação em: 20/07/2007 Aceito para publicação em: 01/12/2009 
ANEXO A - Descrição das variáveis utilizadas por Gee (1990)

\begin{tabular}{|c|c|c|c|}
\hline Símbolo & Descrição & Valor & Unidade \\
\hline$G$ & Concentração de Glicose & 70 (inicial) & $\mathrm{mol} / \mathrm{m}^{3}$ \\
\hline$M$ & Concentração de Maltose & 220 (inicial) & $\mathrm{mol} / \mathrm{m}^{3}$ \\
\hline$N$ & Concentração de Maltotriose & 40 (inicial) & $\mathrm{mol} / \mathrm{m}^{3}$ \\
\hline$X$ & Concentração de Biomassa & 170 & $\mathrm{~mol} / \mathrm{m}^{3}$ \\
\hline$i$ & Açúcar utilizado no parâmetro $(G, M$ ou $N)$ & - & - \\
\hline$m_{1,2 \text { ou } 3}$ & Taxa específica de consumo de açúcar & - & $h^{-1}$ \\
\hline$m_{i}$ & Velocidade máxima de reação para o açúcar " $i$ ” & - & $h^{-1}$ \\
\hline$K_{i}$ & Constante de Michaelis para o açúcar "i” & - & $\mathrm{mol} / \mathrm{m}^{3}$ \\
\hline$K_{i}^{\prime}$ & Constante de inibição para o açúcar " $i$ " & - & $\mathrm{mol} / \mathrm{m}^{3}$ \\
\hline $\ln \mu_{G 0}$ & Fator de frequência de Arrhenius para $V_{G}$ & 35,77 & $\ln h^{-1}$ \\
\hline $\ln \mu_{M 0}$ & Fator de frequência de Arrhenius para $V_{M}$ & 16,4 & $\ln h^{-1}$ \\
\hline $\ln \mu_{N 0}$ & Fator de frequência de Arrhenius para $V_{N}$ & 10,59 & $\ln h^{-1}$ \\
\hline$E_{G 0}$ & Energia de ativação de Arrhenius para $V_{G}$ & 22,6 & kcal/gmole \\
\hline$E_{M 0}$ & Energia de ativação de Arrhenius para $V_{M}$ & 11,3 & kcal/gmole \\
\hline$E_{N o}$ & Energia de ativação de Arrhenius para $V_{N}$ & 7,16 & kcal/gmole \\
\hline $\ln K_{G 0}$ & Fator de frequência de Arrhenius para $K_{G}$ & -121 & $\ln$ gmole $/ \mathrm{m}^{3}$ \\
\hline $\ln K_{M 0}$ & Fator de frequência de Arrhenius para $K_{M}$ & $-19,5$ & ln gmole $/ \mathrm{m}^{3}$ \\
\hline $\ln K_{N 0}$ & Fator de frequência de Arrhenius para $K_{N}$ & $-26,8$ & $\ln$ gmole $/ \mathrm{m}^{3}$ \\
\hline$E_{K G}$ & Energia de ativação de Arrhenius para $K_{G}$ & $-68,6$ & kcal/gmole \\
\hline$E_{K M}$ & Energia de ativação de Arrhenius para $K_{M}$ & $-14,4$ & kcal/gmole \\
\hline$E_{K N}$ & Energia de ativação de Arrhenius para $K_{N}$ & $-19,9$ & kcal/gmole \\
\hline $\ln K_{G 0}^{\prime}$ & Fator de frequência de Arrhenius para $K_{G}^{\prime}$ & 23,33 & $\ln$ gmole $/ \mathrm{m}^{3}$ \\
\hline $\ln K_{M 0}^{\prime}$ & Fator de frequência de Arrhenius para $K_{M}^{\prime}$ & 55,61 & ln gmole $/ \mathrm{m}^{3}$ \\
\hline$E_{K G}^{\prime}$ & Energia de ativação de Arrhenius para $K_{G}^{\prime}$ & 10,2 & kcal/gmole \\
\hline$E_{K M}^{\prime}$ & Energia de ativação de Arrhenius para $K_{M}^{\prime}$ & 26,3 & kcal/gmole \\
\hline$Y_{X G}$ & Fração molar de biomassa por mol de glicose & 0,134 & $\mathrm{~mol} / \mathrm{mol}$ \\
\hline$Y_{X M}$ & Fração molar de biomassa por mol de maltose & 0,268 & $\mathrm{~mol} / \mathrm{mol}$ \\
\hline$Y_{X N}$ & Fração molar de biomassa por mol de maltotriose & 0,402 & $\mathrm{~mol} / \mathrm{mol}$ \\
\hline$K_{x}$ & $\mathrm{C}^{\text {te }}$ de inibição para o crescimento microbiano & 365000 & $\left(\mathrm{~mol} / \mathrm{m}^{3}\right)^{2}$ \\
\hline$E$ & Concentração de etanol & 0 (inicial) & $g / l$ \\
\hline$Y_{E G}$ & Fração molar de etanol por mol de glicose & 1,92 & $\mathrm{~mol} / \mathrm{mol}$ \\
\hline$Y_{E M}$ & Fração molar de etanol por mol de maltose & 3,84 & $\mathrm{~mol} / \mathrm{mol}$ \\
\hline
\end{tabular}




\begin{tabular}{|c|c|c|c|}
\hline$Y_{E N}$ & Fração molar de etanol por mol de maltotriose & 5,76 & $\mathrm{~mol} / \mathrm{mol}$ \\
\hline$C_{L}$ & Concentração de $\mathrm{CO}_{2}$ na fase líquida & 0 (inicial) & $\mathrm{mol} / \mathrm{m}^{3}$ \\
\hline$K_{G L}$ & & 0,07 & - \\
\hline$C_{\text {Sat }}$ & Concentração de saturação de $\mathrm{CO}_{2}$ no líquido & 62,5 & $\mathrm{~mol} / \mathrm{m}^{3}$ \\
\hline$C_{G}$ & Concentração de $\mathrm{CO}_{2}$ na fase gasosa & 0 (inicial) & $\mathrm{mol} / \mathrm{m}^{3}$ \\
\hline$Y_{C G}$ & Fração molar de $\mathrm{CO}_{2}$ por mol de glicose & 1,97 & $\mathrm{~mol} / \mathrm{mol}$ \\
\hline$Y_{C M}$ & Fração molar de $\mathrm{CO}_{2}$ por mol de maltose & 3,94 & $\mathrm{~mol} / \mathrm{mol}$ \\
\hline$Y_{C N}$ & Fração molar de $\mathrm{CO}_{2}$ por mol de maltotriose & 5,76 & $\mathrm{~mol} / \mathrm{mol}$ \\
\hline$V D K$ & Concentração de diacetil & 0 (inicial) & $\mathrm{mol} / \mathrm{m}^{3}$ \\
\hline$Y_{V D K}$ & Fração molar de diacetil por mol de levedura & 0,000105 & $\mathrm{~mol} / \mathrm{mol}$ \\
\hline $\ln k_{V D K}^{0}$ & Fator de frequência de Arrhenius para $\mathrm{K}_{\mathrm{VDK}}^{0}$ & 86,8 & $\ln m^{3} /\left(h^{*} m o l\right)$ \\
\hline$E_{V D K}$ & Energia de ativação de Arrhenius para $\mathrm{k}_{\mathrm{VDK}}^{0}$ & 54,3 & $\mathrm{kcal} / \mathrm{mol}$ \\
\hline$\Delta H_{F G}$ & Calor de reação para a glicose & $-91,2$ & $\mathrm{~kJ} /$ gmole \\
\hline$\Delta H_{F M}$ & Calor de reação para a maltose & -226 & $\mathrm{~kJ} / \mathrm{gmole}$ \\
\hline$\Delta H_{F N}$ & Calor de reação para a maltotriose & -361 & kJ/gmole \\
\hline$\rho_{F}$ & Densidade do mosto & 1040 & $\mathrm{~kg} / \mathrm{m}^{3}$ \\
\hline$\rho_{C}$ & Densidade do fluido refrigerante & 1062 & $\mathrm{~kg} / \mathrm{m}^{3}$ \\
\hline$C p_{F}$ & Calor específico do mosto & 4,016 & $\mathrm{~kJ} / \mathrm{kg} *{ }^{\circ} \mathrm{C}$ \\
\hline$C p_{C}$ & Calor específico do fluido refrigerante & 3,768 & $\mathrm{~kJ} / \mathrm{kg} *{ }^{\circ} \mathrm{C}$ \\
\hline$V_{F}$ & Volume do fermentador & 0,235 & $m^{3}$ \\
\hline$V_{C}$ & Volume da jaqueta de refrigeração & 0,032 & $m^{3}$ \\
\hline$h$ & Coeficiente de troca térmica & 879 & $k J / h * m^{2 * o} C$ \\
\hline$A_{c}$ & Área de contato entre a jaqueta e o fermentador & 1,746 & $m 2$ \\
\hline
\end{tabular}

